\title{
Prevenção quaternária e educação médica: Uma revisão integrativa após as Diretrizes Curriculares Nacionais do Curso Medicina de 2014
}

\author{
Quaternary Prevention and Medical Education: an Integrative Review after National Curricular \\ Guidelines of the Medicine Course of 2014
}

\section{Prevención cuaternaria y educación médica: una Revisión Integrativa después de las Directrices Curriculares Nacionales del Curso Medicina de 2014}

\author{
Carlos Alberto Severo Garcia Jr. ${ }^{1} \oplus$, Amanda Steil ${ }^{1} \odot$, Clara Garcia Miranda ${ }^{1}(0$ \\ ${ }^{1}$ Universidade do Vale do Itajaí (UNIVALI). Itajaí, SC, Brasil.
}

\begin{abstract}
Resumo
Introdução: De acordo com a Resolução ํo 3, de 2014, que institui as Diretrizes Curriculares Nacionais (DCN) do Curso de Graduação em Medicina, o(a) graduando(a) em Medicina deve ter formação geral, humanista, crítica, reflexiva e ética, com capacidade para atuar nos diferentes níveis de atenção à saúde, dentre estes, a prevenção. A investigação diagnóstica deve ser realizada com base nas melhores evidências científicas e conforme as necessidades da pessoa. Nesse sentido, a prevenção quaternária e seus princípios têm relação com os objetivos da DCN. Objetivos: Realizar a intersecção a partir de periódicos científicos brasileiros entre a prevenção quaternária e o ensino médico após a implantação da DCN de 2014. Métodos: Revisão integrativa dos artigos disponíveis nas plataformas da CAPES, Scielo, PubMed e Google Acadêmico, a partir do ano de 2014 até outubro de 2017, por meio das palavras chave "prevenção quaternária" e "educação". Critérios de exclusão: artigos patrocinados por indústrias farmacêuticas, indisponiveis na íntegra, sem resumo e estrangeiros. Resultados: Foram encontrados 33 documentos e 9 artigos selecionados para análise, voltados principalmente para a prática da prevenção quaternária. Apenas 3 estudos fazem alusão à educação médica. Conclusão: A prevenção quaternária é um tema de grande relevância na prática médica, entretanto, ainda é deficiente na graduação, baseado nas restritas produções disponíveis. Apesar da nova DCN do curso de medicina, o ensino médico no cenário atual é predominantemente voltado para especialidades, dificultando a atuação integral e centrada na pessoa.
\end{abstract}

Palavras-chave: Prevenção Quaternária; Educação Médica; Revisão

Como citar: Garcia Jr. CAS, Steil A, Miranda CG. Prevenção quaternária e educação médica: Uma revisão integrativa após as Diretrizes Curriculares Nacionais do Curso Medicina de 2014. Rev Bras Med Fam Comunidade. 2018;13(40):1694. https://dx.doi.org/10.5712/rbmfc13(40)1694

\section{Autor correspondente:}

Carlos Alberto Severo Garcia Jr.

E-mail: carlosgarciajunior@hotmail.com

Fonte de financiamento: declaram não haver.

Parecer CEP:

não se aplica.

Procedência e revisão por pares: revisado por pares.

Recebido em: 21/02/2018.

Aprovado em: 02/01/2019. 


\begin{abstract}
Introduction: According to the Resolution No. 3, 2014, from the Brazilian Ministry of Health, that establishes the National Curricular Guidelines of the Medicine Undergraduate Course. According to the guidelines, the medicine student must have general, humanistic, critical, reflexive and ethical training, with capacity to act at the different levels of health care, among them, prevention. The diagnostic investigation should be based on the best scientific evidence and according to the needs of the person. In this sense, quaternary prevention and its principles meet the objectives of the DNC. Objectives: To perform the intersection in Brazilian journals between the quaternary prevention and medical teaching after the implementation of the DNC of 2014. Methods: Integrative review of the articles available on CAPES, Scielo, PubMed and Google Academic platforms, from 2014 until October 2017, by the keywords "quaternary prevention" and "education". Exclusion criteria includes articles sponsored by pharmaceutical industries, unavailable in their entirety, without abstract and foreign articles. Results: 33 documents were found and 9 articles were selected for analysis, focused mainly on the practice of quaternary prevention. Only 3 studies allude to medical education. Conclusion: Quaternary prevention is a subject of great relevance in medical practice, however, it is still deficient in graduation, based on the restricted productions available. Despite the new guidelines of the medical course, medical education in the current scenario is predominantly focused on specialties, hindering the integral and person-centered performance.
\end{abstract}

Keywords: Quaternary Prevention; Education, Medical; Review

\title{
Resumen
}

Introducción: De acuerdo con las Directrices Curriculares Nacionales (DCN) del Curso de Graduación en Medicina de 2014, el graduando debe tener una formación general, humanista, crítica, reflexiva y ética, con capacidad para actuar en todos los niveles de atención a la salud, entre éstos, la prevención cuaternaria. La investigación diagnóstica debe realizarse sobre la base de las mejores evidencias científicas y según las necesidades de la persona. Además, el médico debe atentar para no perjudicar aquellos que buscan ayuda, causando iatrogenias. En este sentido, la prevención cuaternaria y sus principios tienen relación con los objetivos de la DCN. Objetivos: Buscar activamente la intersección entre la P4 y la educación médica en los periódicos brasileños después de la implantación de la DCN de 2014. Métodos: Revisión integrativa de artículos en las plataformas de CAPES, Scielo, PubMed y Google Académico, a partir del año 2014 hasta octubre de 2017, a través de las palabras-clave "prevención cuaternaria"y "educación". Los criterios de exclusión incluyen los artículos patrocinados por industrias farmacéuticas, indisponibles en su totalidad, sin resumen y extranjeros. Resultados: Se han encontrado 33 documentos y 9 artículos seleccionados para análisis, orientados principalmente a la práctica de la P4. Sólo 3 estudios hacen alusión a la educación médica. Conclusión: La prevención cuaternaria es de gran pertinencia en la práctica médica, sin embargo, todavía es deficiente en la graduación. A pesar de la nueva DCN del curso de medicina, la enseñanza médica es predominantemente orientada a especialidades, dificultando la actuación integral y centrada en la persona.

Palabras clave: Prevención Cuaternaria; Educación Médica; Revisión

\section{Introdução}

No ano de 2014, o Ministério da Educação instituiu, sob a resolução $n^{\circ} 3$, as novas Diretrizes Curriculares Nacionais (DCN) do Curso de Graduação em Medicina. Este documento estabelece princípios, fundamentos e finalidades para a formação em medicina. Segundo a DCN, o(a) graduando(a) em Medicina deve ter formação geral, humanista, crítica, reflexiva e ética, com capacidade para atuar nos diferentes níveis de atenção à saúde, dentre estes, a prevenção. ${ }^{1}$ Durante o processo de promoção de investigação diagnóstica, a DCN refere que a solicitação de exames complementares, por exemplo, deve ser realizada com base nas melhores evidências científicas e conforme as necessidades da pessoa. 
Nesse sentido, a fim de fortalecer a noção de uma medicina pautada nas melhores evidências, apresenta-se a noção da prevenção quaternária (P4), oficializada pela World Organization Family Doctors (WONCA), em 2003, cujos princípios vão de encontro com os objetivos da DCN. ${ }^{2}$ A P4 se baseia na detecção dos indivíduos em risco de tratamentos excessivos, com o objetivo de protegê-los contra intervenções médicas inapropriadas e sugerir alternativas menos danosas. ${ }^{2}$ Para o cuidado clínico ser baseado na P4, faz-se necessária uma abordagem centrada na pessoa, ${ }^{3}$ medicina baseada em evidências e longitudinalidade. ${ }^{4}$

Em uma sociedade que, cada vez mais, preza por uma rápida resolução de todos os seus problemas e sofrimentos, a prática médica acaba acompanhando seus anseios, de modo que a aplicabilidade da P4 se encontra constantemente ameaçada. Não obstante, muitas vezes, a conduta frente à situação trazida ao consultório é, simplesmente, esperar. Não é difícil de compreender por que esse formato de medicina causa estranheza e receio, principalmente por parte da população leiga.

Além disso, uma série de outros elementos, tais como as grandes indústrias farmacêuticas e forte apelo midiático, constroem um terreno fecundo para o sobrediagnóstico e sobretratamento, responsáveis diretamente por uma série de iatrogenias. Segundo Jamoulle e Gomes, ${ }^{5}$ os médicos acabam escondendo suas incertezas por detrás de todo o aparato tecnológico que o mercado hoje oferece, afastando-se do antigo princípio de quem cuida: o de primeiro não causar dano.

É nessa formatação que a educação médica, em geral, prepara seus futuros profissionais. Segundo Gérvas e Pérez-Fernández, ${ }^{6}$ o ensino médico é "centrado na tirania do diagnóstico, no uso da tecnologia, na visão fragmentada do corpo (apenas sem alma) como campo de batalha contra a doença e fator de risco"6 (p. 8).

As universidades são ambientes de formação do pensamento. Consequentemente, refletem diretamente na maneira como o indivíduo irá atuar como médico. Uma das formas de verificar a presença de determinado tema na educação médica dá-se pela análise das produções científicas realizadas acerca do assunto. Assim, o objetivo principal do estudo é analisar de que modo a P4 está presente no meio científico no Brasil, sobretudo após a DCN de 2014, a qual, como salientado previamente, estimula a formação de profissionais com uma postura mais crítica e reflexiva.

Dessa forma, é possível verificar se a P4 é alvo de interesse na formação médica ao visualizar a dimensão de estudos presentes no meio acadêmico-científico, tendo como embasamento diretrizes que apontam para a construção de uma medicina que englobe a totalidade do ser humano e suas reais necessidades evitando ao máximo causar danos.

\section{Métodos}

Para elaboração dos métodos, foram seguidas as recomendações da declaração de Preferred Reporting Items for Systematic Reviews and Meta-Analyses (PRISMA). ${ }^{7}$

Para identificar os artigos que compõem a revisão integrativa foi realizada uma busca ativa das produções científicas brasileiras em bancos de dados na internet. As plataformas utilizadas para a busca foram a biblioteca eletrônica Scientific Electronic Library Online (SciELO), periódicos da Coordenação de Aperfeiçoamento de Pessoal de Nível Superior (CAPES), PubMed e Google Acadêmico. A escolha destes 
meios ocorreu por serem bases de confiabilidade e fácil acesso. Não foi realizado o acesso à base de dados da Literatura Latino-Americana e do Caribe em Ciências da Saúde (LILACS). A última data de acesso aos bancos de dados ocorreu em outubro de 2017.

As estratégias de busca foram definidas por meio dos termos (palavras-chave) "Prevenção Quaternária" e "Educação". Os critérios de inclusão foram estudos brasileiros, disponíveis nas bases de dados descritas, publicados a partir de 2014. Esse recorte temporal se justifica porque é o ano em que foi instituída a nova DCN para a graduação em medicina.

Para a seleção dos artigos, foram aplicados alguns critérios de exclusão, sendo estes: artigos publicados em revistas patrocinadas pela indústria farmacêutica, para retirar o viés de quaisquer conflitos de interesse presentes; artigos sem resumo, visto que encontram-se incompletos e fora das normas padrões para a publicação de artigos; e artigos publicados em revistas estrangeiras e língua inglesa, pois a revisão se propõe a realizar uma intersecção entre a P4 e o ensino médico após a instituição das novas diretrizes curriculares nacionais.

A análise dos resultados obtidos ocorreu pela leitura dinâmica dos títulos e resumos e, após isso, os artigos selecionados foram lidos por completo, verificando os dados que são predominantemente expostos com relação à P4 e as publicações realizadas sobre o tema com enfoque na educação médica. Após obtidos, os dados foram correlacionados com os elementos balizadores propostos pela nova DCN da graduação de medicina.

\section{Resultados}

Por meio dos descritores utilizados "Prevenção Quaternária" e "Educação" foram encontrados 33 artigos e, após a aplicação dos critérios de exclusão e descarte dos artigos duplicados, restaram 9 artigos selecionados para a análise. O diagrama da Figura 1 exemplifica as etapas realizadas no processo da busca.

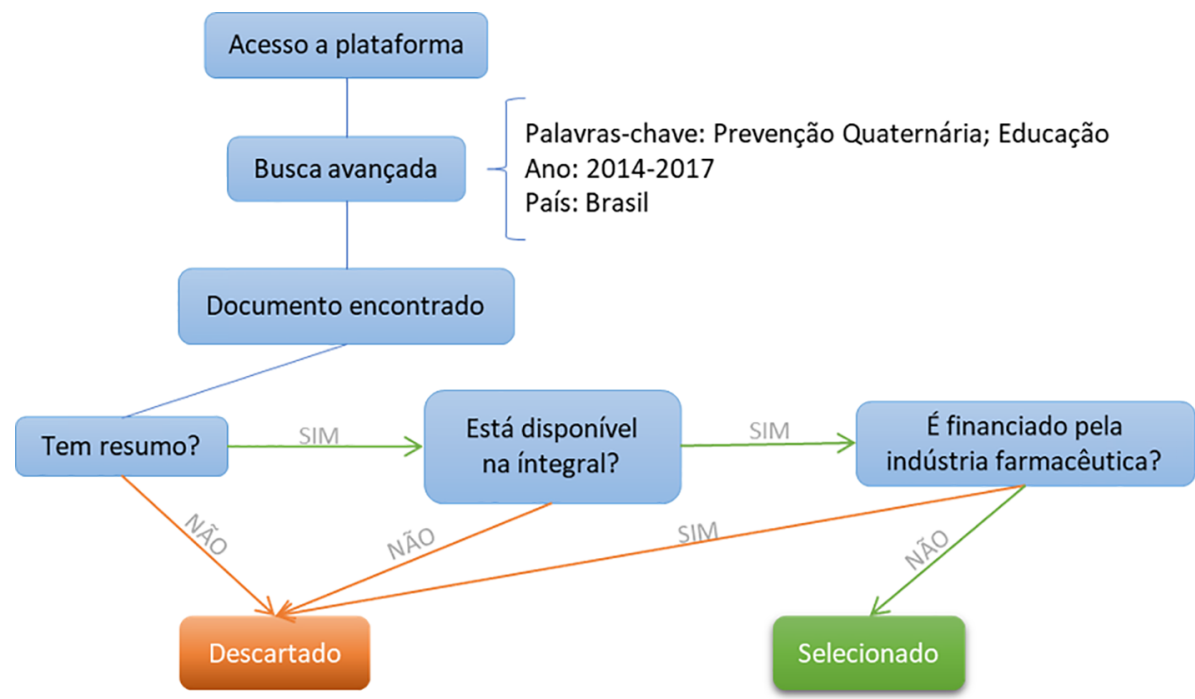

Figura 1. Diagrama de etapas para a busca e seleção dos artigos. Fonte: Autores (2018). 
Na plataforma de periódicos da CAPES, foram aplicados os filtros: "prevenção quaternária" AND "educação"; "2014-2017". Sete artigos foram encontrados; destes, apenas um foi incluído ao estudo, visto que um dos artigos não continha resumo e os cinco restantes não iam de encontro com o tema pesquisado, sendo a P4 citada esporadicamente nos textos.

A busca na biblioteca eletrônica Scientific Electronic Library Online (SciELO) por "Prevenção quaternária" revelou cinco artigos; destes, dois foram selecionados para o estudo. Dois foram descartados, pois seu tema principal não era voltado para a P4 e um dos artigos não possuía resumo. Quando expandida para "Prevenção quaternária" AND "educação", um artigo foi encontrado. Todos os artigos respeitavam o período entre 2014-2017.

No PubMed, “Quaternary Prevention” AND “Education”; 2014-2017, revelou quatro resultados, porém, os autores não eram brasileiros e, portanto, foram excluídos de nosso banco de dados por não versarem nossa realidade.

A busca na plataforma Google Acadêmico foi feita com "prevenção quaternária" no título e "educação" em qualquer parte do texto, foram encontrados 18 resultados no período estabelecido. Destes, foram excluídos cinco artigos sem resumo, quatro artigos estrangeiros, dois resultados que estavam em formato de slides e um artigo não estava disponível na íntegra; sendo assim, seis publicações foram incluídas à revisão.

Os artigos incluídos na revisão sistemática se encontram no Quadro 1, com seus respectivo objetivos e autores, bem como as revistas e ano em que foram publicados.

\section{Discussão}

AP4 é um tema que ainda é pouco trabalhado dentro da graduação médica. A pesquisa revela que o número de publicações voltadas para o assunto disponíveis nas bases de dados nacionais ainda é pequeno. $\mathrm{O}$ cenário do ensino médico foi abordado diretamente em três artigos e apenas dois elaboraram críticas e questionamentos perante a formação dos profissionais. Uma vez que a graduação contém disciplinas e estágios direcionados principalmente para as especialidades, inviabiliza a constituição de um atendimento com integralidade. Os trabalhos selecionados para o estudo foram, em sua maioria, publicações da Revista Brasileira de Medicina de Família e Comunidade (MFC), demonstrando como o tema é interesse predominante dos profissionais da MFC. O alcance da P4 necessita de expansão, visto que deve ser amplamente realizada dentro de todas as áreas de atuação.

Apesar dos critérios de exclusão para a revisão, a busca no PubMed com as palavras chaves "Quaternary Prevention" em título/resumo revelou 60 artigos. Quando acrescida de "Education", os resultados se restringem a apenas 5 artigos, dos quais nenhum deles se correlaciona com o ensino médico na graduação. Isto posto, pode apontar a carência de estudos relacionados a P4 como estratégia de ensino na graduação médica, inclusive no âmbito internacional.

O principal risco desta revisão foi a possibilidade de algum artigo sobre o assunto não ter sido selecionado, uma vez que as palavras chave "Prevenção Quaternária" e "Educação" restringiam a busca. Além disso, a restrição da busca com data após a DCN de 2014, bem como o idioma em português, isenta da revisão artigos de extrema relevância para a P4, desde quando foi conceitualizada. A limitação da língua portuguesa pode, também, ter excluído da seleção artigos sobre o Brasil que foram publicados fora do país. 
Quadro 1. Características dos estudos publicados a respeito da temática (2014-2017).

\begin{tabular}{|c|c|c|c|c|c|c|}
\hline Estudo & Título & Objetivo & Plataforma & Autores & Revista & Ano \\
\hline 1 & $\begin{array}{l}\text { Um novembro não } \\
\text { tão azul: debatendo } \\
\text { rastreamento de câncer } \\
\text { de próstata e saúde do } \\
\text { homem }\end{array}$ & $\begin{array}{l}\text { Discutir o posicionamento } \\
\text { da SBMFC frente a } \\
\text { campanha Novembro Azul } \\
\text { e sua repercussão. }\end{array}$ & Scielo & $\begin{array}{l}\text { Antônio Augusto Dall'Agnol } \\
\text { Modesto, Rodrigo Luciano } \\
\text { Bandeira de Lima, Ana } \\
\text { Carolina D’Angelis, Daniel } \\
\text { Knupp Augusto }\end{array}$ & $\begin{array}{l}\text { Interface, } \\
\text { comunicação, saúde } \\
\text { e educação }\end{array}$ & 2017 \\
\hline 2 & $\begin{array}{l}\text { Diferenciando o cuidado } \\
\text { clínico da prevenção de } \\
\text { doença: um pré-requisito } \\
\text { para praticar a } \\
\text { prevenção quaternária }\end{array}$ & $\begin{array}{l}\text { Discutir a diferenciação } \\
\text { entre cuidado clínico e } \\
\text { prevenção para a prática da } \\
\text { prevenção quaternária. }\end{array}$ & Scielo & $\begin{array}{l}\text { Charles Dalcanale Tesser, } \\
\text { Armando Henrique Norman }\end{array}$ & $\begin{array}{l}\text { Cadernos de Saúde } \\
\text { Pública }\end{array}$ & 2016 \\
\hline 3 & $\begin{array}{l}\text { Prevenção em saúde } \\
\text { na prática médica: da } \\
\text { primária à quaternária }\end{array}$ & $\begin{array}{l}\text { Revisão acerca dos } \\
\text { conceitos de prevenção, } \\
\text { da primária à quaternária, } \\
\text { na literatura em saúde em } \\
\text { língua portuguesa, suas } \\
\text { motivações, definições, } \\
\text { importância, abrangência e } \\
\text { consequência. }\end{array}$ & CAPES & $\begin{array}{l}\text { Tiago Sousa Neiva, Lucy } \\
\text { Gomes Vianna, Clayton } \\
\text { Franco Moraes }\end{array}$ & $\begin{array}{l}\text { Revista Eletrônica } \\
\text { Gestão \& Saúde }\end{array}$ & 2015 \\
\hline 4 & $\begin{array}{l}\text { Violência obstétrica e } \\
\text { prevenção quaternária: o } \\
\text { que é e o que fazer }\end{array}$ & $\begin{array}{l}\text { Justificar a necessidade } \\
\text { de prevenção quaternária } \\
\text { frente à 'violência } \\
\text { obstétrica'. }\end{array}$ & $\begin{array}{c}\text { Google } \\
\text { acadêmico }\end{array}$ & $\begin{array}{l}\text { Charles Dalcanale Tesser, } \\
\text { Roxana Knobel, Halana } \\
\text { Faria de Aguiar Andrezzo, } \\
\text { Simone Grilo Diniz }\end{array}$ & $\begin{array}{l}\text { Revista Brasileira de } \\
\text { Medicina de Família } \\
\text { e Comunidade }\end{array}$ & 2015 \\
\hline 5 & $\begin{array}{l}\text { Prevenção quaternária e } \\
\text { limites em medicina }\end{array}$ & $\begin{array}{l}\text { Descrever a prevenção } \\
\text { quaternária e sua aplicação } \\
\text { na prática. }\end{array}$ & $\begin{array}{l}\text { Google } \\
\text { acadêmico }\end{array}$ & $\begin{array}{l}\text { Marc Jamoulle, Luís Filipe } \\
\text { Gomes }\end{array}$ & $\begin{array}{l}\text { Revista Brasileira de } \\
\text { Medicina de Família } \\
\text { e Comunidade }\end{array}$ & 2014 \\
\hline 6 & $\begin{array}{l}\text { Prevenção quaternária: } \\
\text { as bases para sua } \\
\text { operacionalização na } \\
\text { relação médico-paciente }\end{array}$ & $\begin{array}{l}\text { Apresentar as bases } \\
\text { clínicas e conceituais } \\
\text { para se operacionalizar a } \\
\text { prevenção quaternária na } \\
\text { prática dos serviços de } \\
\text { Atenção Primária à Saúde e } \\
\text { no ambiente de ensino e/ou } \\
\text { programa de residência em } \\
\text { medicina de família }\end{array}$ & $\begin{array}{l}\text { Google } \\
\text { acadêmico }\end{array}$ & $\begin{array}{l}\text { Armando Henrique Norman, } \\
\text { Charles Dalcanale Tesser }\end{array}$ & $\begin{array}{l}\text { Revista Brasileira de } \\
\text { Medicina de Família } \\
\text { e Comunidade }\end{array}$ & 2015 \\
\hline 7 & $\begin{array}{l}\text { Literatura médica e } \\
\text { a ética em pesquisa: } \\
\text { desafios para a } \\
\text { prevenção quaternária }\end{array}$ & $\begin{array}{l}\text { Discutir a qualidade das } \\
\text { evidências científicas } \\
\text { publicadas no campo da } \\
\text { medicina }\end{array}$ & $\begin{array}{l}\text { Google } \\
\text { acadêmico }\end{array}$ & $\begin{array}{l}\text { Hemilton Lima Wagner, Cyn- } \\
\text { thia Ramos Soares, João } \\
\text { Victor Moreira }\end{array}$ & $\begin{array}{l}\text { Revista Brasileira de } \\
\text { Medicina de Família } \\
\text { e Comunidade }\end{array}$ & 2015 \\
\hline 8 & $\begin{array}{l}\text { Prevenção quaternária } \\
\text { na gestão da atenção } \\
\text { primária à saúde: revisão } \\
\text { integrativa }\end{array}$ & $\begin{array}{l}\text { Identificar a Prevenção } \\
\text { Quaternária (PQ) e discutir } \\
\text { suas formas de inserção na } \\
\text { atenção primária à saúde. }\end{array}$ & $\begin{array}{l}\text { Google } \\
\text { acadêmico }\end{array}$ & $\begin{array}{l}\text { Danielle Machado } \\
\text { Portocarrero Gross, } \\
\text { Alessandra Conceição Leite } \\
\text { Funchal Camacho, Leandro } \\
\text { dos Reis Lage, Donizete } \\
\text { Vago Daher, Cristina Portela } \\
\text { da Mota }\end{array}$ & $\begin{array}{l}\text { Rev Enferm UFPE } \\
\text { on line }\end{array}$ & 2016 \\
\hline 9 & $\begin{array}{l}\text { Prevenção Quaternária: } \\
\text { Ética Médica, Avaliação } \\
\text { e Eficiência nos } \\
\text { Sistemas de Saúde }\end{array}$ & $\begin{array}{l}\text { Reunir materiais } \\
\text { produzidos durante VI } \\
\text { Cúpula Ibero-americana } \\
\text { de Medicina de Família } \\
\text { e Comunidade, sobre } \\
\text { alguns temas relacionados } \\
\text { à P4 e estratégias para } \\
\text { difundir o tema no contexto } \\
\text { médico-acadêmico e } \\
\text { populacional. }\end{array}$ & $\begin{array}{l}\text { Google } \\
\text { acadêmico }\end{array}$ & $\begin{array}{l}\text { Miguel Pizzanelli, Marina } \\
\text { Almenas, Robert Quirós, } \\
\text { Carlos Pineda, Esteban } \\
\text { Cordero, Niurka Taureaux, } \\
\text { Maria de Lourdes Rojas, } \\
\text { Karin Kopitowski, Ricardo } \\
\text { La Valle, Monica Nivelo, } \\
\text { Verónica Menares, Agustina } \\
\text { Piñero, Sonia Ortiz, Rolando } \\
\text { Herrera, Alexis Correa Taja }\end{array}$ & $\begin{array}{l}\text { Revista Brasileira de } \\
\text { Medicina de Família } \\
\text { e Comunidade }\end{array}$ & 2016 \\
\hline
\end{tabular}

Fonte: Autores (2018). 
Segundo Neiva et al., ${ }^{8}$ a intervenção médica por meio de ações preventivas possui carências, tais como aumento dos gastos ligados à saúde com a especialização crescente da medicina, o que leva ao desvio da noção de totalidade do paciente através do conhecimento médico voltado para uma ótica puramente biológica; bem como o despreparo da educação médica em formar profissionais que atendam às reais necessidades da população.

Com o intuito de fornecer informações de qualidade para o uso médico, em 1993, foram lançados os fundamentos da Medicina Baseada em Evidências (MBE), que até hoje são utilizados para tomadas de decisões terapêuticas éticas e comprovadas. Entretanto, Wagner et al. ${ }^{9}$ relatam em estudo que a MBE se tornou alvo de indústrias farmacêuticas que, por sua vez, distorcem dados científicos para favorecer o movimento de se obter o lucro a qualquer custo. Esta situação também é comentada por Modesto et al. ${ }^{10} \mathrm{em}$ seu estudo, ao retratar o papel da imprensa em associação com indústria farmacêutica, médicos e grupos de pacientes, que acabam disseminando, por meios midiáticos, uma crescente forma de medicalização: a promoção da doença.

O aumento dos estudos voltados para as afecções, medicações e medidas preventivas levam a pensamentos que revogam a probabilidade do adoecimento. Esta vertente, segundo Norman e Tesser, ${ }^{11}$ estimula a medicalização dos riscos, quer pela introdução na prática clínica de tratamentos e intervenções preventivos, quer pelo sucessivo e progressivo rebaixamento dos pontos de corte diagnósticos e metas para o controle de níveis de pressão arterial, colesterol sérico, hemoglobina glicosilada, Índice de Massa Corporal (IMC), entre outros. Os critérios cada vez mais rigorosos para o estabelecimento de diagnósticos foi criticado por Jamoulle e Gomes ${ }^{5}$ ao colocarem que "somos todos doentes no estágio I se o estágio I for aquele que define a ausência de sintomas para uma afecção predizível” (p. 187). Gross et al. ${ }^{12}$ e Wagner et al., ${ }^{9}$ do mesmo modo, concordam com o exposto em seus respectivos estudos.

Todavia, identificar um fator de risco assintomático ou diagnosticar uma doença em etapas menos avançadas, nem sempre significa menor risco de morrer. De acordo com Modesto et al., ${ }^{10}$ estudos randomizados realizados em vários países demonstraram que a redução da mortalidade é insignificante se comparada aos danos atrelados à aplicação do rastreamento. A medicalização pode reverter situações vitais em quadros patológicos. Como consequência direta, temos a transformação de pessoas sadias em doentes, aumento do consumo de recursos sanitários e falta de controle ao manejar as expectativas da população.

Ao declarar a situação da violência obstétrica, por exemplo, Tesser et al. ${ }^{13}$ apontam para a forte questão cultural como pano de fundo de várias das tomadas de decisões das gestantes, que se mostram, por vezes, permissivas a condutas médicas desnecessárias, pois lhes é culturalmente introjetado um modelo de assistência, oferecido de maneira hegemônica. Pizzanelli et al. ${ }^{14}$ relatam que os pacientes, muitas vezes, impulsionam a medicalização, alheios aos prejuízos expostos, sendo comum encontrarmos presente uma forte demanda "da cura" e a abolição de todo sintoma. As pessoas encontram-se dependentes, incapacitadas para exercer o autocuidado e tolerar o sofrimento e mal-estar. 
Visto os fatores citados anteriormente, a P4 tem por objetivo identificar, justamente, aqueles indivíduos enquadrados nos valores extremos dos critérios que passam a apresentar maiores riscos de sofrer com o processo de supermedicalização.

Desta forma, a P4 serve para protegê-los de uma intervenção médica injustificada, ou seja, iatrogênica ${ }^{10}$. Segundo Jamoulle e Gomes, ${ }^{5}$ em seu cerne, a P4 surge como problematizadora para cada um dos níveis de prevenção em saúde. Ela o faz ao verificar a pertinência da educação para saúde (prevenção primária), questionar sobre se os critérios diagnósticos das doenças e objetivos dos rastreamentos são válidos e verdadeiros (prevenção secundária) e ao pregar mais comunicação com o paciente e a prática de cuidados comprovados por evidência científica (prevenção terciária). ${ }^{5}$

Martins et al., ${ }^{15}$ em estudo publicado em 2018, reveem o conceito de P4, proposto por Jamoulle em 1999, e esclarecem a pertinência de aplicar os princípios da P4 dentro de todos os níveis de prevenção. Redefinem-na como "ações tomada para proteger indivíduos (pessoas, pacientes) de intervenções médicas que podem causar mais dano do que benefício" (p. 107). ${ }^{15}$ Desta forma, é reconhecido que a P4 deve ser uma conduta presente no ínterim de todo atendimento em saúde, reconhecida por todos os profissionais e aplicada de forma individualizada para todos os indivíduos. ${ }^{15}$

AAtenção Primária à Saúde (APS) possui em suas concepções facilidades para a aplicação da P4, pois, para que estes pacientes sejam identificados, é necessário que a abordagem dos problemas leve em conta o tipo de sofrimento da pessoa e sua relação com o tempo. ${ }^{16}$ Jamoulle e Gomes ${ }^{5}$ referem que a P4 é conquistada, sobretudo, por meio de uma relação longitudinal de confiança com o paciente, dispondo do tempo como instrumento. Tal característica se perde na sobreutilização das urgências hospitalares e no acesso livre a todas as especialidades, sendo assim, uma das competências do Médico de Família e Comunidade. $^{5}$

O Médico de Família e Comunidade precisa compreender o aspecto físico da enfermidade, mas também os significados que o processo de adoecimento traz para o paciente e suas famílias. Queixas específicas dentro do eixo anatomopatológico podem exigir medidas medicamentosas e procedimentos, com uma razão risco/benefício favorável. Já situações de sofrimento inespecíficas e situações de adoecimento potencial ou futuro, a razão risco/benefício costuma ser desfavorável e precisa ser pensada de forma crítica baseada nas melhores evidências possíveis. ${ }^{11}$

Para Norman e Tesser, ${ }^{11}$ a prática da P4 pode ser realizada durante todas as consultas, no momento de manejo clínico, onde as decisões idealmente são tomadas em conjunto. Neiva et al. ${ }^{8}$ concordam que a prática médica precisa levar em consideração o paciente como um todo e deve estar fundamentada em um compartilhamento das decisões clínicas com o paciente, desde que, respaldadas em evidências, garantindo um empoderamento da pessoa acerca de sua própria saúde. Em toda intervenção é preciso buscar ativamente aquilo que trará real benefício a esse indivíduo. ${ }^{8}$

Não obstante, a intervenção quaternária só é factível se houver uma relação médico-paciente harmoniosa. ${ }^{11}$ Segundo Gross et al., ${ }^{12}$ o meio mais eficaz de se atingir este nível de prevenção seria: ouvir melhor os pacientes para adaptar o tecnicamente possível ao individualmente necessário e desejado.

Os autores Norman e Tesser ${ }^{11}$ descrevem que uma das medidas de operacionalização da P4 ocorre com o uso criterioso da linguagem. O diálogo precisa ir de encontro com o universo cultural das pessoas envolvidas, para evitar qualquer tipo de desconforto. As crenças, ansiedades e medos do paciente precisam 
ser acolhidos. Caso contrário, os termos escolhidos podem por si só causar iatrogenia (por rotulação e efeito nocebo). Assim, a P4 induz os profissionais a manterem uma proximidade longitudinal e centrarem o cuidado nas pessoas e em suas vivências, protegendo-as de desvios induzidos pelos automatismos da ânsia diagnóstica e terapêutica. ${ }^{11}$ Sendo assim, questiona-se a necessidade de inserir o ensino de técnicas para habilidades em comunicação dentro da graduação, aprimorando a sensibilidade do acadêmico para identificar a melhor linguagem a ser utilizada com o paciente a fim de atingir a compreensão e evitar iatrogenias.

Visto isso, a P4 torna-se conceito fundamental no atendimento e, como efeito secundário desejável, leva a uma redução considerável dos custos dos atendimentos na APS. ${ }^{12}$

Para que seja exercida com parcimônia, a P4 impõe uma estrita necessidade de o profissional estar atualizado sobre os estudos científicos de boa qualidade voltados para avaliar a relação risco-benefício dos rastreamentos, o que significa que precisa usar a MBE, inexoravelmente, para bem embasar, técnica e eticamente, sua decisão de rastrear. ${ }^{12}$ Ademais, é necessária uma avaliação criteriosa da literatura científica antes de realizar a tomada de decisões. ${ }^{7}$

Em relação à educação, poucos estudos se relacionaram com o ensino médico. Norman e Tesser ${ }^{11}$ comentam que as discussões pautadas para a prática da P4 devem ser relevantes para os profissionais, as instituições de saúde, mas também para disciplinas relacionadas à saúde em cursos de graduação e pós-graduação. Segundo Gross et al., ${ }^{12}$ a formação dos profissionais está voltada para especialidades médicas, e, consequentemente, direciona o raciocínio clínico para o rastreamento de doenças específicas das especialidades.

O olhar amplo necessário para a realização de uma APS de qualidade muitas vezes encontra-se deficiente. Assim, o diagnóstico "especializado" inviabiliza a realização de um diagnóstico preciso, pois não há espaço para os atendimentos longitudinais para que este seja definido com certeza, como também, as vulnerabilidades do território, o histórico social, a relação familiar e outros fatores que podem estar inseridos no processo saúde doença de cada indivíduo não são levados em consideração. ${ }^{12}$ A graduação deve estimular um olhar social e político, a fim de incluir os determinantes sociais no desenvolvimento de políticas de saúde e no exercício próprio da profissão. ${ }^{14}$

De acordo com Gross et al. ${ }^{12}$ e Pizzanelli et al. ${ }^{14}$ o ensino da P4 é uma estratégia que deve ser disseminada permanentemente no Sistema Único de Saúde (SUS) e na formação dos profissionais de saúde, para que a APS seja exercida com excelência na Estratégia de Saúde da Família, diminuindo a medicalização e a iatrogenia do cuidado.

Em seu estudo, Gross et al. ${ }^{12}$ vai de encontro com a novas DCNs ao relatar a importância de formar profissionais que já saiam para o mercado de trabalho com uma postura holística, a qual lhes permite entender o SUS em todas as suas linhas de ação.

Um único estudo publicado na Revista Brasileira de Medicina de Família e Comunidade, em 2015, por Gomes et al., ${ }^{17}$ discorre sobre o ensino e a aprendizagem da P4. Demonstra técnicas para ensino e aprendizagem da P4 que podem ser aplicadas na formação acadêmica e profissional, bem como as competências que devem ser desenvolvidas em cada nível (iniciante, competente, proficiente e expert). Não cabe neste artigo esmiuçar o roteiro proposto pelos autores, porém, é necessário salientar que este é o único estudo que trabalha explicitamente a P4 na educação médica. Todavia, apesar de estar publicado 
na Revista Brasileira de Medicina de Família e Comunidade, por estar em língua estrangeira não pode ser englobado nos resultados da presente revisão.

Gomes et al. ${ }^{17}$ afirmam que ensinar prevenção quaternária é um processo complexo e desafiante que envolve habilidades de comunicação, relação médico-paciente e, sobretudo, uma abordagem centrada no aluno, em que o educador sai do papel de apenas transmitir informações e se torna um facilitador que guia as experiências do próprio aluno. Para que a P4 seja globalmente utilizada, como sugerem Martins et al., ${ }^{15}$ aspectos "biopsicossociais" e holísticos devem estar integrados ao conhecimento do contexto econômico, dos serviços de saúde e da tecnologia incorporada no contexto em que se apresenta. ${ }^{17}$

Segundo Pizzanelli et al., ${ }^{14}$ em âmbito populacional, deve-se estabelecer um modelo de informação mais direto em todos os meios midiáticos possíveis: panfletos, cartazes, avisos em rádios, televisivos, redes sociais, sites, web, blogs, vídeos, de modo a estimular os pacientes a conhecer suas opções, prós e contras.

Nesse contexto, a P4 mostra-se uma ferramenta fundamental não só na APS, mas em todo o SUS, pois ao eliminar os tratamentos e rastreamentos diagnósticos desnecessários, assim como a sobremedicalização, os custos com saúde do sistema tendem a diminuir, assim, possibilitando um melhor uso dos recursos disponíveis no cenário econômico atual. ${ }^{14}$

Conforme apontado por Norman e Tesser, ${ }^{18}$ o ensino da prevenção quaternária é um ponto estratégico para uma prática em APS de excelência. Deve ocorrer no contexto de formação dos profissionais de saúde e ser relembrado regularmente com blocos de educação permanente no SUS. No intuito de que a prática se consolide para diminuir a medicalização e a iatrogenia do cuidado, ainda relativamente pouco percebida no Brasil, segundo os autores.

Sendo assim, a revisão realizada apresenta como benefícios contribuir para a comunidade acadêmica, demonstrando os trabalhos realizados após as mudanças curriculares e, de certa forma, constatar se os resultados encontrados se espelham ou se contrastam à educação médica atual.

\section{Conclusões}

O presente estudo, a partir das buscas realizadas, identifica uma predominância de artigos publicados na Revista Brasileira de Medicina de Família e Comunidade. O tema da P4 demonstrou-se, portanto, ser uma preocupação encontrada em profissionais e estudiosos envolvidos com a APS. A revisão bibliográfica evidenciou que poucos artigos englobam a graduação médica, porém, não podemos concluir se o assunto é pouco discutido dentro das instituições ou se há pouco interesse dos acadêmicos e docentes em realizar pesquisas sobre o tema.

Sendo assim, sugere-se que outras modalidades de pesquisa sejam utilizadas para verificar a presença da P4 na graduação, por meio de análise do currículo ou entrevistas com os próprios acadêmicos, podendo averiguar se as competências relacionadas a inserção da P4 estão presentes nos profissionais docentes e se este conhecimento está no cerne das práticas clínicas diárias, principalmente no internato médico, onde o ensino é centralizado nas experiências do aluno e há uma relação mais próxima com o paciente. 
Apesar dos assuntos abordados nos estudos voltados para a P4 serem equivalentes com a nova DCN da graduação de medicina, ao indicarem o atendimento individualizado com escolhas terapêuticas e diagnósticas embasadas em evidências, nenhuma pesquisa até o momento citou o documento da DCN em si.

A P4 é um tema de grande relevância dentro da prática clínica dos profissionais e pode trazer como resultados a diminuição da medicalização, de equívocos diagnósticos e de ações preventivas que não trazem benefícios reais aos pacientes.

Dentro da premissa da P4, é de suma importância que haja fomento para produções científicas pelas instituições governamental e ensino já na graduação, estimulando o aprendizado sobre o tema para formar profissionais aptos a um atendimento integral e singular, respeitando os limites das intervenções médicas desnecessárias.

\section{Contribuição dos autores}

Concepção do estudo: AS, CGM, CGJ; Aquisição e análise dos dados: AS, CGM; Redação preliminar: AS, CGM; Revisão crítica da versão preliminar: CGJ; Submissão do estudo: CGJ; Cumprimento das alterações solicitadas pelo Conselho Editorial da RBMFC: AS, CGM, CGJ; Revisão da redação final: CGJ.

Todos os autores aprovaram a versão final e concordaram prestar contas sobre todos os aspectos do trabalho.

\section{Agradecimentos}

Agradecemos à Liga de Medicina de Família e Comunidade da UNIVALI pela oportunidade de termos um espaço em que foi possível o encontro dos autores, com o subsequente desejo de realizar o presente estudo.

\section{Conflito de interesses}

Declaram não haver.

\section{Referências}

1. Brasil. Ministério da Educação. Conselho Nacional de Educação. Resolução № 3, de 20 de junho de 2014. Institui as Diretrizes Curriculares Nacionais do Curso de Graduação em Medicina e dá outras providências. Brasil. Ministério da Educação; 2014 . [acesso 2017 Out 2017 16]. Disponível em: http://www.fmb.unesp.br/Home/Graduacao/resolucao-dcn-2014.pdf

2. Bentzen N. An international glossary for general/family practice. Fam Pract. 1995 [acesso 2017 Out 16];12(3):341-69. https://dx.doi. org/10.1093/fampra/12.3.341

3. Stewart M, Brown JB, Weston WW, McWhinney IR, McWilliam CI, Freeman TR. Medicina centrada na pessoa: transformando o método clínico. 2ª ed. Porto Alegre: Artmed; 2010.

4. Starfield B. Atenção Primária: equilíbrio entre necessidades de saúde, serviços e tecnologia. Brasília: Ministério da Saúde/UNESCO; 2002.

5. Jamoulle M, Gomes LF. Prevenção Quaternária e limites em medicina. Rev Bras Med Fam Comunidade. 2013 [acesso 2017 Out 16];9(31):186-91. https://dx.doi.org/10.5712/rbmfc9(31)867 
6. Gérvas J, Pérez-Fernández M. São e salvo: E Livre de Intervenções Médicas Desnecessárias. Porto Alegre: Artmed; 2016.

7. Moher D, Liberati A, Tetzlaff J, Altman DG; PRISMA Group. Preferred reporting items for systematic reviews and meta-analyses: the PRISMA statement. PLoS Med. 2009 [acesso 2018 Jan 14];6(7):e1000097. https://dx.doi.org/10.1371/journal.pmed.1000097

8. Neiva TS, Vianna LG, Moraes CF. Prevenção em saúde na prática médica: da primária à quaternária. Rev Eletr Gestão Saúde. 2015 [acesso 2017 Out 16];6(2):1418-27. https://dx.doi.org/10.18673/gs.v6i2.22477

9. Wagner HL, Soares CR, Moreira JV. Literatura médica e a ética em pesquisa: desafios para a prevenção quaternária. Rev Bras Med Fam Comunidade. 2015 [acesso 2017 Out 16];10(35):1-4. https://dx.doi.org/10.5712/rbmfc10(35)1116

10. Modesto AAD, Lima RLB, D’Angelis AC, Augusto DK. Um novembro não tão azul: debatendo rastreamento de câncer de próstata e saúde do homem. Interface (Botucatu). 2018 [acesso 2017 Out 16];22(64):251-62. https://dx.doi.org/10.1590/1807-57622016.0288

11. Norman AH, Tesser CD. Prevenção quaternária: as bases para sua operacionalização na relação médico-paciente. Rev Bras Med Fam Comunidade. 2015 [acesso 2017 Out 16];10(35):1-10. https://dx.doi.org/10.5712/rbmfc10(35)1011

12. Gross DMP, Camacho ALCF, Lage LR, Daher DV, Mora CP. Prevenção quaternária na gestão da atenção primária à saúde: revisão integrativa. Rev Enferm UFPE On Line (Recife). 2016 [acesso 2017 Out 16];10(Supl. 4):3608-19. Disponível em: https://periodicos.ufpe. br/revistas/revistaenfermagem/article/download/11136/12633. DOI: 10.5205/reuol.9681-89824-1-ED.1004sup201613

13. Tesser CD, Knobel R, Andrezzo HFA, Diniz SG. Violência obstétrica e prevenção quaternária: o que é e o que fazer. Rev Bras Med Fam Comunidade. 2015 [acesso 2017 Out 16];10(35):1-12. https://dx.doi.org/10.5712/rbmfc10(35)1013

14. Pizzanelli M, Almenas M, Quirós R, Pineda C, Cordero E, Taureaux N, et al. Prevenção Quaternária: Ética Médica, Avaliação e Eficiência nos Sistemas de Saúde. Rev Bras Med Fam Comunidade. 2016 [acesso 2017 Out 16];11(Suppl 2):75-85. https://dx.doi.org/10.5712/ rbmfc11(0) 1388

15. Martins C, Godycki-Cwirko M, Heleno B, Brodersen J. Quaternary prevention: reviewing the concept. Eur J Gen Pract. 2018 [acesso 2018 Dez 2];24(1):106-11. https://dx.doi.org/10.1080/13814788.2017.1422177

16. Tesser CD, Norman AH. Differentiating clinical care from disease prevention: a prerequisite for practicing quaternary prevention. Cad Saúde Pública. 2016 [acesso 2017 Out 16];32(10):e00012316. https://dx.doi.org/10.1590/0102-311X00012316

17. Gomes LF, Gusso G, Jamoulle M. Teaching and learning quaternary prevention. Rev Bras Med Fam Comunidade. 2015 [acesso 2018 Dez 2];10(35):1-14. https://dx.doi.org/10.5712/rbmfc10(35)1050

18. Norman AH, Tesser CD. Quaternary prevention in primary care: a necessity for the Brazilian Unified National Health System. Cad Saúde Pública. 2009 [acesso 2018 Dez 2];25(9):2012-20. https://dx.doi.org/10.1590/S0102-311X2009000900015 